\title{
Dependence of the structure of core-shell-corona micelles on the composition of water/toluene mixtures
}

\author{
Liangcai Lei ${ }^{\text {a,1 }}$ Jean-François Gohy ${ }^{\text {a, } 2}$, Nicolas Willet ${ }^{a}$, Jian-Xin Zhang ${ }^{b}$, Sunil Varshney ${ }^{b}$, Robert Jérôme ${ }^{a}$ \\ ${ }^{a}$ Institute of Chemistry B6, Centre for Education and Research on Macromolecules (CERM), University of Liège, Sart-Tilman, B-4000 \\ Liège, Belgium \\ ${ }^{\mathrm{b}}$ Polymer Source, 124 Avro, Dorval, Que., Canada H9P 2X8
}

\begin{abstract}
The poly(styrene)-block-poly(2-vinylpyridine)-block-poly(ethylene oxide) triblock copolymer, $\mathrm{PS}_{200}-b$ - $\mathrm{P}_{2} \mathrm{VP}_{140^{-}}$ $b$ - $\mathrm{PEO}_{590}$, where the subscripts refer to the average degrees of polymerisation of the constitutive blocks, has been dissolved in water/toluene mixtures with a large range of composition, i.e. in (i) toluene added with a small amount of water, (ii) water-in-toluene emulsion forming mixtures, (iii) toluene-in-water emulsion forming mixtures, and (iv) water added with a small amount of toluene. These solutions have been cast on a carboncoated copper grid, and the morphology of the dried copolymer deposition has been observed by transmission electron microscopy. Rod-like aggregates with a core-shell-corona micellar structure are formed in cases (i) and (iv). Nevertheless, PEO is the core and PS is the corona in case (i), whereas the reverse situation prevails in case (iv). When an emulsion is the precursor of the dried copolymer aggregates, either onion-like structures (case ii) or vesicles mixed with more complex aggregates are formed (case iii). These structures are thought to reflect the self-organization of the $\mathrm{PS}_{200}-b-\mathrm{P}_{2} \mathrm{VP}_{140}-b-\mathrm{PEO}_{590}$ copolymer at the water/toluene interface of the water-intoluene and toluene-in-water emulsions, respectively.
\end{abstract}

Keywords: Triblock copolymers; Micelles; Morphological transition

\section{Introduction}

Micelles with tailored shape and size are receiving a rapidly growing attention as templates for the production of well-defined nanoobjects.

Eisenberg and coworkers have pioneered the control of micellar morphologies, which basically depends on three key parameters, i.e. (i) stretching of the core-forming chains, (ii) core-corona interfacial energy and (iii) repulsion between the coronal chains. These parameters have a direct impact on the Gibbs free energy of the micelles, as exemplified by the morphological transitions displayed by the so-called crew-cut micelles formed by highly asymmetric block copolymers that contain a major insoluble core-forming block [1]. Indeed, Eisenberg and coauthors were able to trigger these morphological transitions not only by changing the copolymer composition [2] but also by adding salts [3] or non-selective cosolvents [4]. Although diblock copolymers are the most popular promoters of micelles, ABC triblock copolymers deserve interest because of the higher complexity of the generated nanostructures [5,6]. A typical example may be found in formation of spherical core-shellcorona (CSC) micelles by poly(styrene)-block-poly(2-vinylpyridine)-block-poly(ethylene oxide) (PS- $b$-P2VP- $b$ PEO) triblock copolymers in water [7,8]. These micelles consist of a PS core, a pH-responsive shell and a PEO corona. Although the range of the structural characteristic features of these micelles can be increased by changing the length and composition of the PS- $b$-P2VP- $b$-PEO copolymers, a more direct strategy can rely on the use of cosolvents, as assessed by a sphere-to-rod morphological transition triggered by the addition of a small amount of toluene to water [9]. This transition is the answer of the micelles to the selective swelling of the coreforming PS chains by toluene. Indeed, an increase in the volume fraction of the core-forming blocks of spherical micelles results in an entropy penalty, such that a morphological sphere-to-rod transition is observed beyond a critical volume fraction [1,2]. Until now, the rod-like micelles have been collected and studied in water after

\footnotetext{
${ }^{1}$ On leave from the Department of Applied Chemistry, Liaoning University of Petroleum and Chemical Technology, Fushun, People's Republic of China.

${ }^{2}$ Present address: Unité de Chimie des Matériaux Inorganiques et Organiques (CMAT), Département de Chimie, Université catholique de Louvain.
} 
dialysis of toluene [9]. This study aims at preparing PS- $b$-P2VP- $b$-PEO micelles in water/toluene mixtures of various toluene contents and at investigating the impact of the swelling of the PS core on the micellar morphology.

\section{Experimental part}

\subsection{Micelles preparation}

The $\mathrm{PS}_{200}-b-\mathrm{P} 2 \mathrm{VP}_{140}-b-\mathrm{PEO}_{590}$ triblock copolymer (the numbers in subscript being the average degree of polymerisation of each block) was synthesized by living anionic polymerization with a polydispersity index of 1.1, as reported elsewhere [7]. It was dissolved in (i) toluene containing a small amount of water, (ii) water-intoluene emulsion forming mixtures, (iii) toluene-in-water emulsion forming mixtures and (iv) water containing a small amount of toluene, respectively. In case (i), $0.01 \mathrm{~g}$ of $\mathrm{PS}_{200}-b$-P2 $\mathrm{VP}_{140}-b-\mathrm{PEO}_{590}$ was dissolved into $10 \mathrm{ml}$ of dried toluene $(C=1 \mathrm{~g} / \mathrm{L})$. Then, various amounts of water were dropwise added under vigorous stirring that was maintained for $48 \mathrm{~h}$. When the water content exceeded $0.10 \mathrm{vol} \%$, a milky solution was formed as a typical water-in-toluene emulsion (case ii). At water content higher than $60 \mathrm{vol} \%$, the inverse emulsion was observed (case iii). Micelles $(C=1 \mathrm{~g} / \mathrm{L})$ in case (iv) were prepared as reported elsewhere [7]. Reverse micelles formed in toluene-rich mixture (less than 0.1 vol\% water) were cross-linked by reaction of the P2VP chains with 1,4diiodobutane, molar ratio $=1 / 2$ ) under stirring at room temperature for $72 \mathrm{~h}$. The cross-linked micelles were insoluble in THF at room temperature (milky solution) in contrast to the uncross-linked material that formed a clear solution under the same conditions.

\subsection{Transmission electron microscopy (TEM)}

TEM images were recorded with a Philips CM100 microscope equipped with a Gatan 673 CCD camera, and transferred to a computer equipped with the Kontron KS100 system. Samples were prepared by casting a drop of the micellar solution onto a carbon-coated TEM grid and contrasted with $\mathrm{RuO}_{4}$ vapor. Samples were also stained by a phosphotungstic acid aqueous solution. In this case, a drop of $0.1 \mathrm{wt} \%$ phosphotungstic acid aqueous solution was deposited onto the surface of the sample-loaded grid. Three minutes later, the solution excess was removed with a filter paper, and the sample was washed with water and dried in air. The standard deviation for the characteristic sizes measured by TEM was $3 \mathrm{~nm}$ for all the pictures.

\section{Results and discussion}

A previous paper has shown that the addition of a selective solvent for the PS block of the $\mathrm{PS}_{200}-b-\mathrm{P}_{2} \mathrm{VP}_{140}-b$ $\mathrm{PEO}_{590}$ copolymer was able to trigger a morphological sphere-to-rod transition in the micelles formed in water [9]. Fig. 1 is a typical TEM picture of the accordingly formed rod-like micelles. The PS core is easily discriminated from the P2VP shell by the proper use of selective staining agents. Indeed, $\mathrm{RuO}_{4}$ selectively stains both the PS core and the P2VP shell, whereas phosphotungstic acid is a selective staining agent for the P2VP shell. As a result, the characteristic diameter of the PS core plus the P2VP shell, $D_{\mathrm{CS}}$, the diameter of the PS core, $D_{\mathrm{C}}$, and the thickness of the P2VP shell, $H_{\mathrm{S}}$, can be directly determined from the TEM pictures of the dried micelles. All these data are reported in Table 1 . The thickness of the corona, $H_{\mathrm{Co}}$, was also determined from a TEM picture, whose the background was negatively stained and the PS and P2VP phases were contrasted by $\mathrm{Ru}_{4}$ [9]. These rod-like micelles can be observed in water/ toluene mixture containing less than 0.1 vol\% of toluene (case iv).

Whenever the $\mathrm{PS}_{200}-b-\mathrm{P}_{2} \mathrm{VP}_{140}-b$ - $\mathrm{PEO}_{590}$ copolymer is dissolved in pure toluene, no micellization occurs as assessed by the solution analysis by dynamic light scattering. Consistently, no micelle is observed when the solution is cast on a carbon-coated TEM grid, rather a film is formed with a typical lamellar morphology. Nevertheless micellization occurs as soon as $\sim 0.03$ vol\% of water is added to a $1 \mathrm{~g} / \mathrm{L}$ copolymer solution in toluene. Actually, this critical amount of water is very close to the limit of solubility of water in toluene at room temperature [10], which might indicate that micellization is driven by the formation of water microphases and the migration of the triblock copolymer towards the toluene/water interface in order to minimize the interfacial tension. It must, however, be noted that the copolymer solution in toluene remains optically clear until the water content in toluene is $\sim 0.10 \mathrm{vol} \%$. Interestingly enough, this water content is increased up to $0.15 \mathrm{vol} \%$ when the copolymer concentration in water is two times higher $(2 \mathrm{~g} / \mathrm{L})$. These observations give credit to the hypothesis that the primary role of water is to solvate the PEO blocks by hydrogen-bonding interactions. A rough calculation indicates that there is approximately one water molecule per PEO unit when the $1 \mathrm{~g} / \mathrm{L}$ solution of $\mathrm{PS}_{200}-b$-P2 $\mathrm{VP}_{140}-b-\mathrm{PEO}_{590}$ copolymer is added with 0.03 vol\% water. No water microphase would thus be formed at this stage. Rather, the hydrated PEO blocks would have a strong tendency to self-assemble in toluene, 
with formation of micelles consisting of a hydrated PEO core, a P2VP shell and a PS corona. At a 0.1 vol\% water in the $1 \mathrm{~g} / \mathrm{L}$ copolymer solution, all the hydrogen-bonding sites of the PEO blocks appear to be saturated by water, that phase separates and makes the solution turbid. Because the swollen PEO blocks are the major component (higher volume fraction) in the triblock, micelles should be non-spherical. This expectation is confirmed by TEM observation of rod-like micelles in the dried state (Fig. 2). These rods which are regularly branched, actually consist of a P2VP shell, as emphasized by the selective staining by phosphotungstic acid (Fig. 2(a)). In Fig. 2(b), both the PS and P2VP blocks have been stained by $\mathrm{RuO}_{4}$, which accounts for a thicker shaded layer compared to Fig. 2(a). Comparison of Figs. 1(b) and 2(b) shows the reversed structures for the rod-like micelles which are formed in water-rich (case iv) and in toluene-rich (case i) toluene/water mixtures, respectively. The central PS core of the rods is stained by $\mathrm{RuO}_{4}$ in the water-rich system, whereas the PEO core is not contrasted by this agent in the toluene-rich one. Because micelles have been observed by TEM after drying, it is essential to know whether the micellar morphology resists drying or not. Therefore, reverse micelles have been cross-linked by reaction of the P2VP chains with 1,4-diiodobutane (cf. Section 2). Although soluble in $\mathrm{THF}$ at room temperature (clear solution), the triblock copolymer formed a milky solution under the same conditions, as result of effective cross-linking. No significant change in shape and size of the micelles is observed upon cross-linking, which strongly suggests that the morphologies herein reported are representative of the situation in solution. This experiment has been conducted with reverse micelles, because, the content of water may be lower than $0.1 \mathrm{vol} \%$, which is a prerequisite for the cross-linking reaction to be successful.

The stretching of the PS blocks $S_{\mathrm{PS}}$ ) was estimated as the ratio of $H_{\mathrm{Co}}$ (i) or the core radius (ii) to the unperturbed end-to-end distance of the PS chains.

Fig. 1. Rod-like aggregates of $\mathrm{PS}_{2} \mathrm{Oo}-\mathrm{e}-\mathrm{P} 2 \mathrm{VPi} \mathrm{i}_{4} \mathrm{o}-\mathrm{e}-\mathrm{PE} \mathrm{P} 59 \mathrm{o}$ formed in water-rich toluene/water mixtures and stained by phosphotungstic acid ( $a$, selective for $P 2 V P$ ) or $R u O_{4}$ vapor $(b$, selective for both $P S$ and $P 2 V P)$. A scheme of these structures is shown in (c).
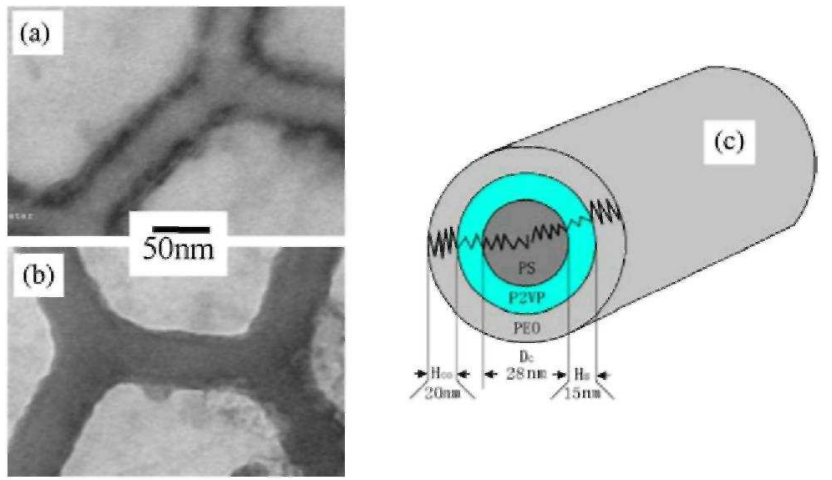

Table 1 : Morphological characteristic features of rod-like CSC micelles prepared either in toluene-rich or in water-rich mixtures

\begin{tabular}{lllllll}
\hline $\begin{array}{l}\text { Toluene/water } \\
\text { mixtures }\end{array}$ & Micellar structure & $\boldsymbol{D}_{\mathbf{C s}}{ }^{\mathbf{a}}$ & $\boldsymbol{D}_{\mathbf{C}}{ }^{\mathbf{a}}$ & $\boldsymbol{H}_{\mathbf{S}}{ }^{\mathbf{a}}$ & $\boldsymbol{H}_{\mathbf{C o}}{ }^{\mathbf{a}}$ & $\boldsymbol{S}_{\mathbf{P S}}{ }^{\mathbf{b}}$ \\
\hline Toluene-rich & $\begin{array}{l}\text { Core (PEO)-shell } \\
\text { (P2VP)-corona }\end{array}$ & 32 & 14 & 9 & 9 & 0.96 \\
Water-rich & $\begin{array}{l}\text { (PS) } \\
\text { Core (PS)-shell } \\
\text { (P2VP)-corona } \\
\text { (PEO) }\end{array}$ & 58 & 28 & 15 & 20 & 1.5 \\
& & & & & & \\
\hline
\end{tabular}

${ }^{\mathrm{a}} D_{\mathrm{CS}}\left(\right.$ shell diameter), $D_{\mathrm{C}}$ (core diameter), $H_{\mathrm{S}}$ (shell thickness) and $H_{\mathrm{Co}}$ (corona thickness) as determined from TEM pictures (nm). 
Fig. 2. Rod-like aggregates of $P S_{200}-b-P 2 V P_{140}-b-P E O_{590}$ formed in toluene-rich toluene/water mixtures and stained by phosphotungstic acid (a) selective for $\mathrm{P} 2 \mathrm{VP}$ ) or $\mathrm{RuO}_{4}$ vapor (b) selective for both $\mathrm{PS}$ and $\left.\mathrm{P} 2 \mathrm{VP}\right)$. A scheme of these structures is shown in $(c)$.
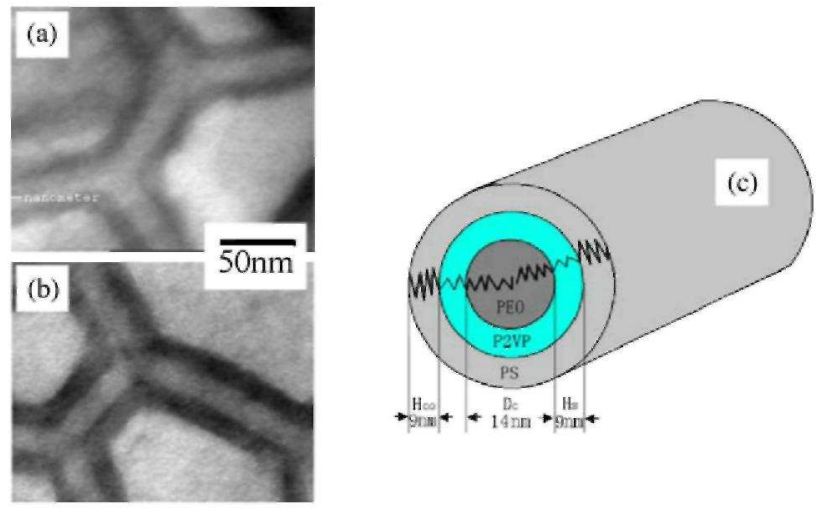

The characteristic sizes of the constitutive compartments of the two types of rod-like micelles have been determined from the TEM pictures and compared in Table 1. It must be kept in mind that these dimensions are characteristic of dried samples and do not reflect the actual situation in solution. Nevertheless, several pertinent conclusions can be drawn. The thickness of the corona, $H_{\mathrm{Co}}$, is smaller in the toluene-rich system, in agreement with the shorter PS block ( $\mathrm{DP}=200)$ compared to the PEO block ( $\mathrm{DP}=590)$ that forms the corona in the waterrich system. Surprisingly enough, the thickness of the P2VP shell, $H_{\mathrm{S}}$, is smaller $(9 \mathrm{~nm})$ for the toluene-rich system than for the water-rich one $(15 \mathrm{~nm})$. A similar observation has, however, been reported for spherical micelles formed by $\mathrm{PS}_{200}-b-\mathrm{P}_{2} \mathrm{VP}_{140}-b-\mathrm{PEO}_{590}[7,8]$. Indeed, the conformation of the $\mathrm{P} 2 \mathrm{VP}$ chains in the shell changes from a random-coil conformation $\left(H_{\mathrm{S}} \approx 7.5 \mathrm{~nm}\right)$ in a non-solvent for PEO to a stretched conformation in case of PEO hydration $\left(H_{\mathrm{S}} \approx 15 \mathrm{~nm}\right)$. By analogy, the P2VP chains in the shell would be rather in a random coil conformation in the toluene-rich system and in an extended one in the water-rich solvent mixture. Before proposing a tentative explanation of this morphological characteristic feature, it must be noted that the diameter of the core, $D_{\mathrm{C}}$, is smaller in case of the toluene-rich system although the degree of polymerization of the coreforming PEO block (590) is much larger than the $\mathrm{PS}_{200}$ core in the water-rich system. These amazing observations can find their origin in a difference in the organization of the core-forming chains. Indeed, the PS chains are stretched in the core of the micelles in the water-rich system, as confirmed by the degree of stretching $\left(S_{\mathrm{PS}}\right)$ estimated as the ratio of the actual size $\left(H_{\mathrm{Co}}\right.$ or $\left.D_{\mathrm{C}}\right)$ to the unperturbed end-to-end distance of the PS chains (Table 1). A regular packing of the stretched PS chains is a reasonable organization which is schematized in Fig. 3(a) and results in the stretching of the adjacent P2VP segments. When the micellization free energy is concerned, the entropy loss associated to the stretching of both the PS and the P2VP blocks would be counterbalanced by the exothermicity of the hydrogen-bonding of the PEO coronal chains and water molecules. The situation is completely different when hydrated PEO cores are formed in the toluene-rich system. The micellization free energy is then dominated by the enthalpic contribution of the hydrogen-bonds between the PEO core and water, so preventing the PEO chains from being regularly aligned in a stretched conformation. The adjacent P2VP chains would accordingly adopt a random coil conformation, as schematically shown in Fig. 3(b), so accounting for a $D_{\mathrm{C}}$ value smaller in the toluene-rich system compared to the water-rich one.

Whenever more than 0.10 vol\% of water are added to the $1 \mathrm{~g} / \mathrm{L}$ copolymer solution in toluene, a milky emulsionlike mixture is formed and stabilized by the preferential location of the ABC copolymer at the water/toluene interface. The PEO blocks should point to the water phase and the PS and P2VP blocks should be extended in the toluene phase. This simplified picture is at least partly confirmed by the large single-walled and multi-walled (onions) vesicular structures observed by TEM (Fig. 4). Similar vesicular structures are observed in toluene with a water content in the $0.10-50 \mathrm{vol} \%$ range.

At water content higher than $50 \mathrm{vol} \%$, the morphology changes completely upon drying the water/toluene mixtures on a TEM grid (Fig. 5(a) and (b)). This morphology, referred to as 'coral snake' aggregates, has never been observed until now for micellar systems at least to the best of our knowledge, although it was previously reported in the bulk [11-13]. It consists of a rod-like core surrounded by regularly spaced rings. Because this morphology is observed in water-rich solvent mixtures, the central rod would contain the PS blocks swollen by toluene and would be wrapped by rings of P2VP stained by $\mathrm{RuO}_{4} \mathrm{PEO}$ cannot be identified when $\mathrm{RuO}_{4}$ is the staining agent. Vesicles are commonly observed at the extremities of the 'coral snake' structures, as shown in Fig. 5(a) and (b). It thus appears that the 'coral snake' morphology is a transient structure than can be trapped when 
core-shell-corona micelles go through a rod-to-vesicle transition. Actually, vesicles coexist with the 'coral snake' aggregates as shown in Fig. 5(c) and (d).

Fig. 3. Schematic chain conformation in each compartment of the rod-like aggregates in water-rich (a) and in toluene-rich (b) water/toluene mixtures, respectively.

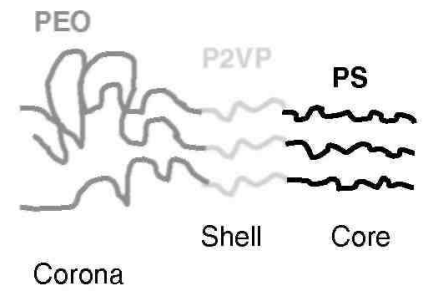

(a)

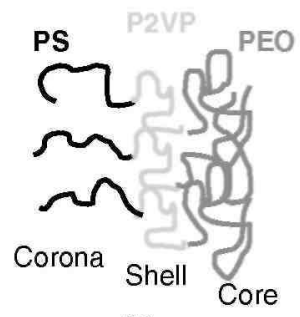

(b)

Fig. 4. TEM image of onion-like vesicles formed in water-in-toluene emulsions (staining by $\mathrm{RuO}_{4}$ ).

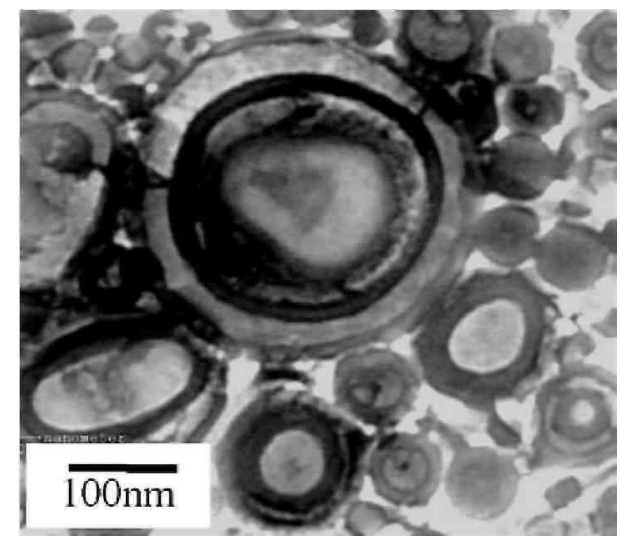

Fig. 5. TEM images of 'coral snake' aggregates ( $a$ and $b$ ) and vesicles ( $c$ and $d$ ) formed in toluene-in-water emulsions (staining by $\mathrm{RuO}_{4}$ ).
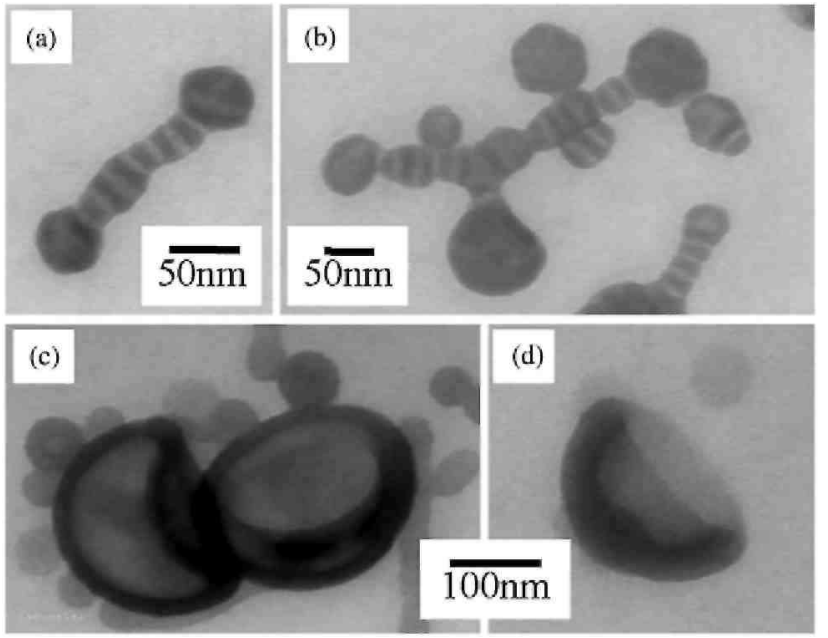


\section{Conclusions}

Quite interestingly, a single $\mathrm{PS}_{200}-b-\mathrm{P} 2 \mathrm{VP}_{140}-b-\mathrm{PEO}_{590}$ triblock copolymer can form aggregates with different morphologies in water/toluene mixtures of various compositions. The sequence of micellar structures observed in this study can be tentatively rationalized by the toluene content of the solvent mixtures. In pure water, spherical CSC micelles are formed [7]. At low content of toluene, the shape of the micelles changes from spherical to cylindrical while maintaining a PS core, a continuous P2VP shell and a PEO corona [9]. At higher toluene content, the shorter P2VP chains are facing problem in wrapping homogeneously the swollen PS rod, which results in discontinuous rings of P2VP characteristic of the so-called 'coral snake' aggregates. As soon as the PS core cannot accommodate anymore higher amount of toluene, droplets of toluene are dispersed in water, and the copolymer organizes itself at the water/toluene interface, so leading to vesicular structures.

In order to establish the actual relationships between these structures and the solvent mixture composition, the micelles and/or their aggregates should be stabilized by cross-linking before deposition and drying on the TEM grids. This strategy was successfully used in the specific case of reverse micelles. However, cross-linking reaction effective in water-rich micellar solutions must be searched for. If not successful, cryo-TEM might be a valuable alternative.

\section{Acknowledgements}

The authors are very much indebted to 'Belgian Science Policy' for financial support in the frame of the 'Interuniversity Attraction Poles Programme (PAI V/03) - Supramolecular Chemistry and Supramolecular Catalysis'. JFG is grateful to the ESF STIPOMAT Programme.

\section{References}

[1] Zhang L, Eisenberg A. Science 1995;268:1728.

[2] Zhang L, Eisenberg A. J Am Chem Soc 1996;118:3168.

[3] Zhang L, Eisenberg A. Science 1996;272:1777.

[4] Discher DE, Eisenberg A. Science 2002;297:967.

[5] Goldacker T, Abetz V, Stadler R, Erukhimovich I, Leibler L. Nature 1999;398:137.

[6] Stupp SI, LeBonheur V, Walker K, Li LS, Huggins KE, Keser M, et al. Science 1997;276:384.

[7] Gohy J-F, Willet N, Varshney S, Zhang J-X, Jérôme R. Angew Chem Int Ed 2001;40:3214.

[8] Gohy J-F, Willet N, Varshney SK, Zhang J-X, Jérôme R. e-Polym 2002;35.

[9] Lei L, Gohy J-F, Willet N, Varshney SK, Zhang J-X, Jérôme R. Macromolecules 2004;37:1089.

[10] Dean JA. Analytical chemistry handbook. New York: McGraw-Hill Inc.; 1995 p. 2-20.

[11] Stadler R, Auschra C, Beckmann J, Krappe U, Voigt-Martin I, Leibler L. Macromolecules 1995;28:3080.

[12] Breiner U, Krappe U, Abetz V, Stadler R. Macromol Chem Phys 1997; 198:1051.

[13] Abetz V, Jiang S, Göpfert A. e-Polym 2004;40. 